\title{
Supplementary
}

\section{Chiral Protein Supraparticles for Tumor Suppression and Synergistic Immunotherapy - an enabling strategy for bioactive supramolecular chirality construction}

Jin $\mathrm{Yan}^{1}$, $\mathrm{Yu} \mathrm{Yao}{ }^{2}$, Siqi $\mathrm{Yan}^{3}$, Ruqing $\mathrm{Gao}^{4}$, Wuyuan $\mathrm{Lu}^{5}$ and Wangxiao $\mathrm{He}^{6,7}$

1. National \& Local Joint Engineering Research Center of Biodiagnosis and Biotherapy, The Second Affiliated Hospital, Xi'an Jiaotong University, Xi'an, China.

2. Department of Medical Oncology, The First Affiliated Hospital of Xi'an Jiaotong University.

3. Ophthalmology Department, The First Affiliated Hospital of Xi'an Jiaotong University, Xi'an 710061, China.

4. School of Medicine, Nanchang University, Nanchang 330006, China.

5. Institute of Human Virology and Department of Biochemistry and Molecular Biology, University of Maryland School of Medicine, Baltimore, MD 21201, USA.

6. Department of Talent Highland, The First Affiliated Hospital of Xi'an Jiao Tong University, Xi' an 710061, China.

7. The Second Affiliated Hospital of Xi'an Jiao Tong University, Xi'an 710061, China.

$\dagger$ These authors contributed equally.

${ }^{*}$ Corresponding authors:

Email:hewangxiao5366@xjtu.edu.cn (W.He);

Email:wlu@ihv.umaryland.edu (W.Lu);

Email:yanjin19920602@xjtu.edu.cn (J. Yan) 


\section{Experimental Section}

\section{General remarks}

All synthetic peptide sources were obtained from CS Bio (Shanghai) Ltd. All other chemicals used in this study were purchased from Sigma-Aldrich unless otherwise specified. Acetonitrile and water (HPLC grade) were purchased from Fisher Scientific Ltd. All products were used as received without further purification.

\section{Synthesis of ${ }^{\mathrm{D}} \mathrm{MSN},{ }^{\mathrm{D}} \mathrm{Apamin}$ and ${ }^{\mathrm{D} P M I^{\beta}}$}

All peptides were synthesized on appropriate resins on an CS bio 336X automated peptide synthesizer using the optimized HBTU activation/DIEA in situ neutralization protocol developed by an HBTU/HOBt protocol for Fmoc-chemistry SPPS. 2 After cleavage and deprotection in a reagent cocktail containing $88 \%$ TFA, $5 \%$ phenol, $5 \% \mathrm{H}_{2} \mathrm{O}$ and $2 \%$ TIPS, crude products were precipitated with cold ether and purified to homogeneity by preparative C18 reversed-phase HPLC. The molecular masses were ascertained by electrospray ionization mass spectrometry (ESI-MS).

We prepared DMSN, DApamin and Apamin by orthogonally protecting Cys3 and Cys 15 with Acm first, followed by oxidative folding of the peptide with two free Cys residues (Cys1 and Cys11). The intermediate with one native disulfides was treated with iodine at acidic $\mathrm{pH}$ to spontaneously deprotect and oxidize Acm-protected Cys3 and Cys15. ${ }^{\mathrm{D}}$ Apamin and Apamin were synthesized as the same protocol as DMSN.

\section{CD spectroscopy.}


CD spectra of ${ }^{\text {DMSN }}$ variants at a concentration of $20 \mu \mathrm{M}$ in $10 \mathrm{mM}$ phosphate buffer ( $\mathrm{pH}$

7.4) were obtained at room temperature on a J-810 spectropolorimeter (Jasco, Easton, MD) using a 1-mm quartz cuvette. Scanned area was from $250 \mathrm{~nm}$ to $190 \mathrm{~nm}$, and the scanning speed was 50 $\mathrm{nm} / \mathrm{min}$. Every curve was the average of three independent detection.

\section{Proteolytic stability}

proteolytic resistance of ${ }^{\mathrm{D}} \mathrm{MSN}$ and its complete enantiomer ${ }^{\mathrm{L}} \mathrm{MSN}$ were incubated at $100 \mu \mathrm{M}$ each in PBS with $20 \mu \mathrm{g} / \mathrm{ml}$ cathepsin $\mathrm{G}$ - an intracellular protease with dual specificities for both basic and bulky hydrophobic residues- in GSH free and 10mM GSH buffer. RP-HPLC was used to monitor and quantify time-dependent peptide hydrolysis.

\section{Fluorescence polarization-based competitive binding assay}

As for fluorescence polarization (FP) assay, Fluorescein (FITC) was conjugated to ${ }^{15-29} \mathrm{p} 53$

via its N-terminal amino group in DMF, and the resultant product ${ }^{15-29} \mathrm{p} 53$-FITC were HPLCpurified and lyophilized. The Fluorescence polarization-based competitive binding assay were performed in Microfluor ${ }^{\circledR}$ 2, 96-well black plates (Thermo Fisher Scientific) and readings were taken using a Tecan Infinite M2000 fluorescence plate reader. Serially diluted ${ }^{\text {DMSN }}$ or ${ }^{D}$ PMI ${ }^{\beta}$ were prepared in Tris- $\mathrm{HCl}$ buffered saline (10 $\mathrm{mM}$ Tris, $150 \mathrm{mM} \mathrm{NaCl}, 1 \mathrm{mM}$ EDTA, $\mathrm{pH}$ 7.0), to which $10 \mathrm{nM}{ }^{5-29}$ p53-FITC and $50 \mathrm{nM}$ MDM2 or 500nM MDMX were added in a total volume of $150 \mu \mathrm{L}$ per well. After a 2-h incubation at room temperature, fluorescence polarization was measured at $\lambda \mathrm{ex}$ $=470 \mathrm{~nm}$ and $\lambda \mathrm{em}=530 \mathrm{~nm}$. Nonlinear Curve Fit were performed by Logistic function to give rise to IC50 values. 


\section{Quantification of cellular uptake of ${ }^{\mathrm{D}} \mathrm{MSN}$}

The cellular uptake of ${ }^{\mathrm{D}} \mathrm{MSN}$ was detected using confocal laser scanning microscopy (CLSM, FV1200, Olympus) and flow cytometer (BD Biosciences, NJ). ${ }^{\mathrm{DMSN}}$, ${ }^{\mathrm{D}} \mathrm{Apamin}$ and ${ }^{\mathrm{DPMI}}{ }^{\beta}$ were first re-dispersed in culture medium at a concentration of $50 \mu \mathrm{g} / \mathrm{mL}$. HCT116 cells were cultured in growth medium without drug for $24 \mathrm{~h}$. The culture medium was then replaced with the growth medium containing FITC-labelled ${ }^{\mathrm{D}} \mathrm{MSN},{ }^{\mathrm{D}} \mathrm{Apamin}$ or ${ }^{\mathrm{D}} \mathrm{PMI}{ }^{\beta}$ at $\mathrm{pH} 7.4$, and cultured at $37^{\circ} \mathrm{C}$ for 6 h. Cell imaging or flow cytometry analysis were then carried out after washing the cells with PBS twice to remove the excess drug. For cell imaging, Hoechst33342 (Molecular probes) was used as the controls. To prevent cell falls from the bottom, we used paraformaldehyde to fix cells before observation. For confocal fluorescence microscopy imaging, the excitation was provided by the continuous wave laser at $405 \mathrm{~nm}(3.15 \mathrm{~mW})$ and $543 \mathrm{~nm}(0.7 \mathrm{~mW})$, respectively.

\section{Cell culture and cell viability analysis}

Human colon cancer cell line HCT116 $6^{+/}$(wild-type p53) was purchased from ATCC, and maintained in McCoy's 5A medium with 10\% FBS. The isogeneic HCT116 ${ }^{-/}$(p53 deletion) cells were presented by Prof. Bert Vogelstein of Johns Hopkins University (Baltimore, MD), and maintained in McCoy's 5A medium with 10\% FBS. Melanoma cell line A-375, human hepatoma cell line Sk-Hep-1, breast cancer cell line MCF-7 and human were also purchased by ATCC, and maintained in DMEM with $10 \%$ FBS. All cells were maintained at $37^{\circ} \mathrm{C}$ in an atmosphere of $5 \%$ $\mathrm{CO}_{2}$. Cells were plated in $96-$ well plates at a density of 2500 cells/well $(100 \mu \mathrm{l})$. After $24 \mathrm{~h}$, cells were treated with ${ }^{\mathrm{D}} \mathrm{MSN},{ }^{\mathrm{D}} \mathrm{Apamin},{ }^{\mathrm{D} P M I}{ }^{\beta}$ or Nutlin3 at the indicated concentrations and times, 
respectively. The in vitro cytotoxicity was then measured by using a standard MTT (Thermo Fisher scientific) assay.

\section{Western blot analysis}

Cells were treated with ${ }^{D}$ MSN, ${ }^{D}$ Apamin, ${ }^{\text {DPMI }}{ }^{\beta}$ or Nutlin3 for $24 \mathrm{~h}$, respectively. Cells were then lysed in prechilled RIPA buffer containing protease inhibitors, and equal amounts of protein lysates were separated by $10 \%$ SDS-PAGE and transferred onto PVDF membranes (Roche Diagnostics, Mannheim, Germany). The membranes were subsequently incubated with the indicated primary antibodies at $4{ }^{\circ} \mathrm{C}$ overnight. Antibodies against p53 and p 21 were purchased from Abcam. Antibody against $\beta$-actin was purchased from Sigma-Aldrich. This was followed by incubation with their respective HRP-conjugated secondary antibodies from Calbiochem, and immunoblotting signals were visualized using the Western Bright ECL detection system (Advansta, CA). The results of relative protein level were analyzed using Image $\mathrm{J}$.

\section{Apoptosis and cell cycle analysis}

Necrosis/apoptosis was evaluated by flow cytometric analysis using the FITC Annexin V Apoptosis Detection Kit (BD Biosciences). Briefly, cells were treated with ${ }^{\mathrm{D} M S N}$, ${ }^{\mathrm{D} A p a m i n},{ }^{\mathrm{D} P M}{ }^{\beta}$ or Nutlin 3 for $48 \mathrm{~h}$. Cells were then harvested, washed twice with cold PBS, and re-suspended in $1 \times$ binding buffer at a concentration of $1 \times 10^{6}$ cells $/ \mathrm{ml}$. One hundred microliters of the solution $(1$ $\times 10^{5}$ cells) was transferred to a $5 \mathrm{ml}$ culture tube, followed by addition of $5 \mu 1$ of FITC Annexin $\mathrm{V}$ and $5 \mu 1$ of PI. After gentle vortexing and a 15-min incubation in the dark at room temperature, 400 $\mu 1$ of $1 \times$ binding buffer was added to the tube, and cells were analyzed by FACS. 
Cells were first serum starved for $12 \mathrm{~h}$, and then treated with ${ }^{\mathrm{D} M S N},{ }^{\mathrm{D} A p a m i n},{ }^{\mathrm{D} P M I}{ }^{\beta}$ or Nutlin3 for $24 \mathrm{~h}$. Next, cells were harvested, washed twice in PBS, and fixed in 70\% ethanol on ice for at least $30 \mathrm{~min}$. After that, cells were stained with propidium iodide (PI) solution (50 $\mu \mathrm{g} / \mathrm{ml} \mathrm{PI}$, $50 \mu \mathrm{g} / \mathrm{ml}$ RNase A, $0.1 \%$ Triton-X, $0.1 \mathrm{mM}$ EDTA). Cell cycle distributions were then analyzed based on DNA contents by a flow cytometer (BD Biosciences, NJ).

\section{In vivo bio-distribution analysis}

HCT116 cells $\left(4 \times 10^{6}\right.$ cells/site $)$ were implanted subcutaneously into hip of four- to fiveweeks-old male athymic nude mice. Four weeks after cell inoculation, tumor-bearing mice were injected with $200 \mu \mathrm{L}$ Cy3-labeled ${ }^{\mathrm{D} M S N}$, Cy3-labeled ${ }^{\mathrm{L}} \mathrm{MSN}$ and Cy3-labeled ${ }^{\mathrm{DPMI}}{ }^{\beta}$ at a dosage of $1.5 \mathrm{mg} / \mathrm{ml}$. Of note, all fluorescent samples were prepared by co-incubating peptide with Cyanine3 NHS ester at a molar ratio of 1:1. IVIS Spectrum In Vivo Imaging System was then used to take the ex vivo images of mice. Light with a wavelength at $530 \mathrm{~nm}$ was used as the excitation source and the $620 \mathrm{~nm}$ as the absorption. In addition, mice were humanely killed at predetermined times, and the heart, liver, spleen, lung, kidney and tumor were immediately collected from each mouse. The fluorescence intensity in all organs was further analyzed by the IVIS Spectrum In Vivo Imaging System.

\section{HCT116 xenografts tumor modle.}

All experimental procedures involving animals were conducted in accordance with Institution

Guidelines and were approved by the Laboratory Animal Center of Xi'an Jiaotong University. Tumor cells were harvested when they reached near confluence by incubation with $0.05 \%$ trypsin- 
EDTA. Cells were pelleted by centrifugation and resuspended in sterile PBS. HCT116 cells $(4 \times$ $10^{6}$ cells/site) were implanted subcutaneously into hip of four- to five-weeks-old male athymic nude mice. When the tumors reached average volume of $\sim 50 \mathrm{~mm}^{3}$, the mice were randomly divided into different groups (five mice per group), and treatment was initiated. Tumor length and width were measured with calipers, and tumor volume was calculated using the following equation: tumor volume $(V)=$ length $\times$ width $^{2} / 2$. For histological examination, the tumor, liver, kidney, heart, spleen and lung tissues were fixed with formaldehyde, dehydrated, sliced into $5.0 \mu \mathrm{m}$ sections and subjected to H\&E or immunohistochemical staining. Besides, blood routine examinations (white blood cells, red blood cells and thrombocyte), liver function detections (liver weight, the activities of aspartate aminotransferase (AST) and alanine transaminase (ALT)), kidney function detection (blood urea nitrogen(BUN), serum creatinine(CRE)), and some biochemical enzyme indexes were carried out at the Clinical Laboratory, the First Affiliated Hospital of Xi'an Jiaotong University, according to the standard clinical laboratory procedures.

\section{Colon-cancer-patient-derived xenograft tumor.}

At the time of primary tumor reductive surgery, a specimen was cut into about $5 \mathrm{~mm}$ pieces and implanted into the fossa iliaca of NOD/SCID mice aged $4 \sim 5$ weeks. Volume of tumor was calculated as the above equation in HCT116 xenograft tumor. When the tumors reached approximately 150 $\mathrm{mm}^{3}$, mice were administrated intraperitoneally at a dosage of $1.5 \mathrm{mg} / \mathrm{kg}$ every other day. Xenografts were collected for formalin-fixed-paraffin embedding (FFPE), snap frozen in liquid nitrogen or subsequently implanted into another set of mice using the same procedure. The animal study was approved by the Animal Research Committee of The First Affiliated Hospital of Xi'an Jiao Tong University and helped by the Suzhou Jima gene co. LTD. 
B16F10 metastatic-like lung tumors. We collected and resuspended B16F10 cells in 1640 medium without FBS. $1 \times 10^{5}$ cells per $0.2 \mathrm{~mL}$ cells were then injected into the vein tail of C57BL/6 mice. Drug injections were started 5 th day after cell injection at $1.5 \mathrm{mg} / \mathrm{kg}$ every other day for consecutive 1 week ( 4 times injection). The mice were euthanized on the 15 th day to compare therapy efficacy between different administrations.

\section{Immunohistochemical (IHC) staining}

Sections were cut at $5 \mu \mathrm{m}$ thickness, deparaffinized and rehydrated. Endogenous peroxidase activity was blocked with hydrogen peroxide/methanol, and antigen retrieval was performed in a pH 9.0 TE (Tris-EDTA) buffer by autoclave for $10 \mathrm{~min}$. The resultant tissue sections were then incubated with the antibodies against MDM2, MDMX, p53, p21, and Ki67 at $4^{\circ} \mathrm{C}$ overnight. After incubation with labeled streptavidin-biotin (LSAB) complex for 15 min, the slides were stained and visualized by using the iView DAB detection system (ZSGB-BIO, P.R. China). Each stained section was evaluated by a minimum of 10 randomly selected $\times 20$ high-power fields for further statistical analysis.

To evaluate immunostaining intensity (I), we used a numeric score ranging from 0 to 3 , reflecting the intensity as follows: 0 , no staining; 1 , weak staining; 2, moderate staining; and 3, intense staining. To evaluate immunostaining area (A), we used a numeric score ranging from 1 to 4, reflecting the intensity as follows: 1 , positive area $<10 \% ; 2,10 \%<$ positive area $<50 \% ; 3$, $50 \%<$ positive area $<90 \%$; and 4, positive area $>90 \%$. Using an Excel spreadsheet, the mean score was obtained by multiplying the intensity score (I) by the percentage of positive area and the results were added together (total score: $\mathrm{I} \times \mathrm{A}$ ). 
2. Supplementary figures 


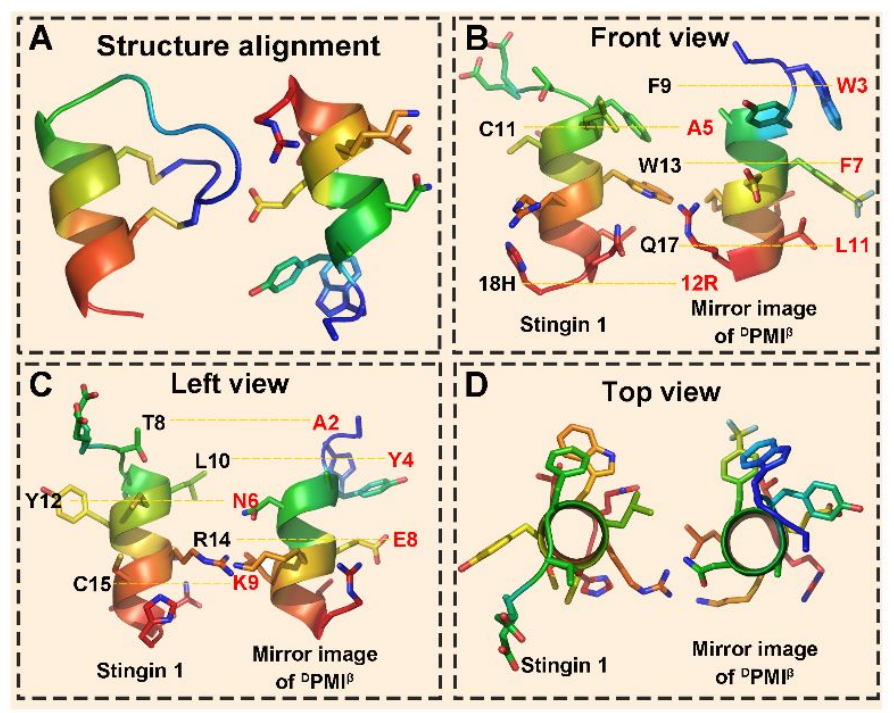

Figure S1. The structural alignment of ${ }^{D} P M I^{\beta}$ and Apamin. A, the structural alignment between Apamin and the L-enantiomer of ${ }^{D P M I}{ }^{\beta}$, which was converted from the structure of ${ }^{D P M I}{ }^{\beta}$ (PDB: 3IWY) via flipping with Cartesian coordinates along the $\mathrm{x}$ axis. $\mathrm{B}-\mathrm{D}$, the detailly structural alignment between the mirror image of ${ }^{\mathrm{DPMI}}{ }^{\beta}$ and an Apamin derived miniprotein, termed Stingin 1. Due to the deficiency of the crystal structure of Apamin and it has been proved that Stingin 1 was consistent with the known structural features of Apamin ${ }^{[1]}$, we compared the mirror image of ${ }^{\mathrm{DPMI}}{ }^{\beta}$ to Stingin 1 on front view (B), left view (C) and top view (D). 

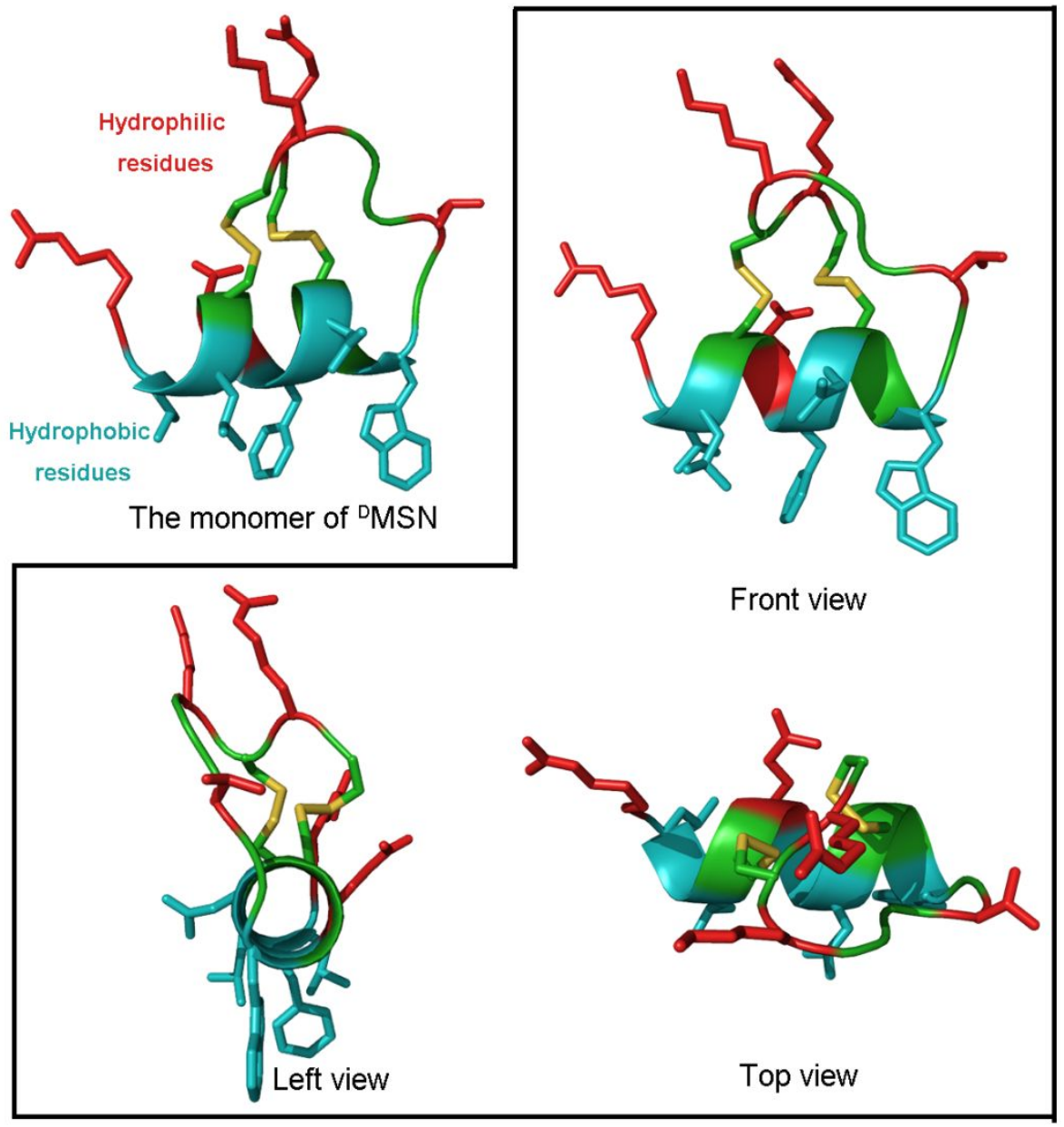

Figure S2. The structure diagram of the monomer of ${ }^{\mathrm{D}} \mathrm{MSN}$. The hydrophobic residues were colored in red, and the hydrophilic residues were colored in cyan. It can be found that hydrophobic side chains and hydrophilic side chains were respectively in the two flanks of the monomer of DMSN. 
A

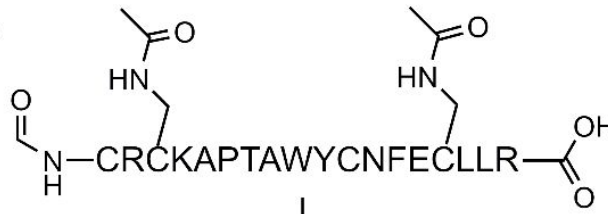

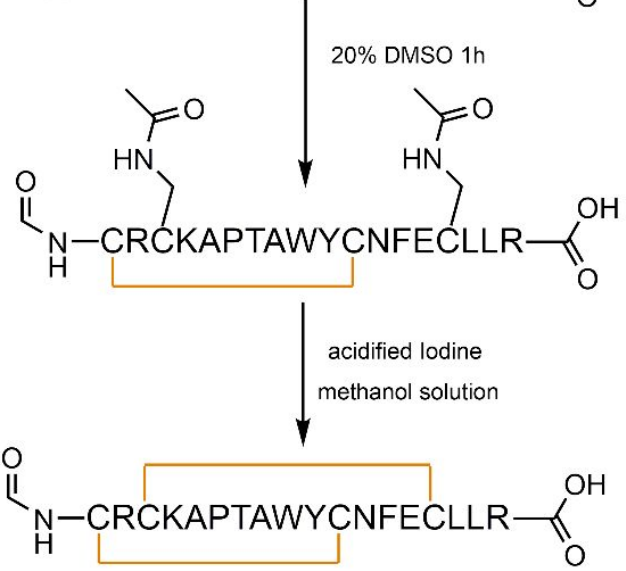

$\underbrace{Y \mathrm{O}}_{\mathrm{HN}}$ Acetaminomethy (ACM)

Disulfide bond

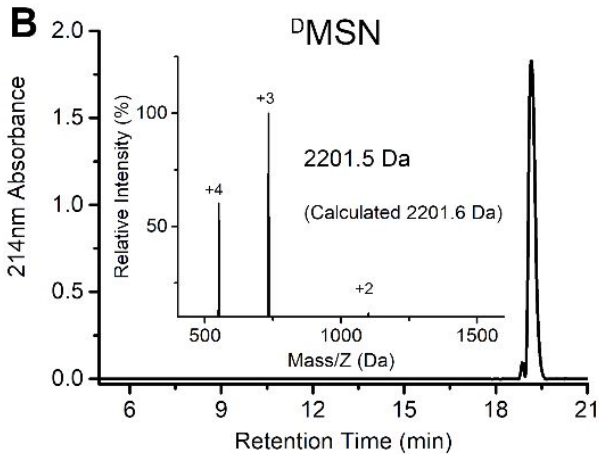

Figure S3. The synthesis of ${ }^{\mathrm{D} M S N}$ monomer. A, the schematic diagram for the disulfide bond generation of ${ }^{\mathrm{D}} \mathrm{MSN}$ via a two-step oxidation. B, the High Performance Liquid Chromatography (HPLC) and ESI-MASS results of DMSN, suggesting that it was successful synthesized. 


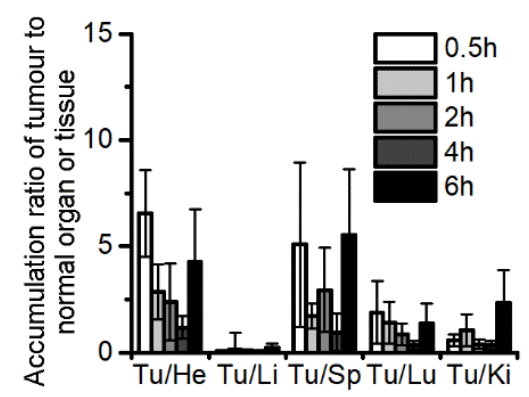

Figure S4. Tumor-to-normal tissue ratios for cy3-labled ${ }^{\mathrm{D}} \mathrm{PMI}{ }^{\beta}$ after intraperitoneal injection at $0.5,1,2,4$ and $6 \mathrm{~h}$. 


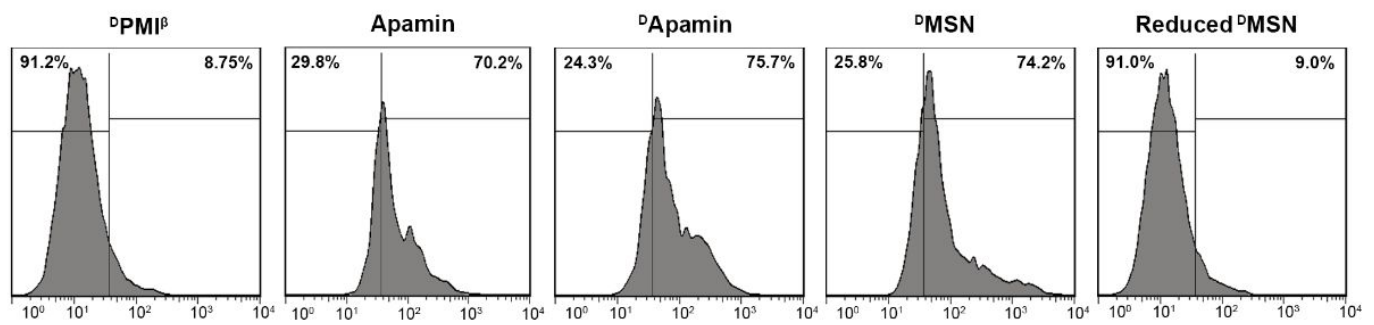

Figure S5. HCT116 cells were incubated with ${ }^{\mathrm{L}} \mathrm{Apamin}{ }^{\mathrm{FITC}}$, ${ }^{\mathrm{D}} \mathrm{Apamin}{ }^{\mathrm{FITC}}$, DMSN ${ }^{\mathrm{FITC}}$, unfolded${ }^{D}$ MSN ${ }^{\text {FITC }}$ and ${ }^{D P M I}{ }^{\beta-F I T C}$ at the concentration of $200 \mu \mathrm{g} / \mathrm{ml}$ for $6 \mathrm{~h}$. Cellular uptake was then measured by flow cytometry analysis via the green fluorescence from FITC. 


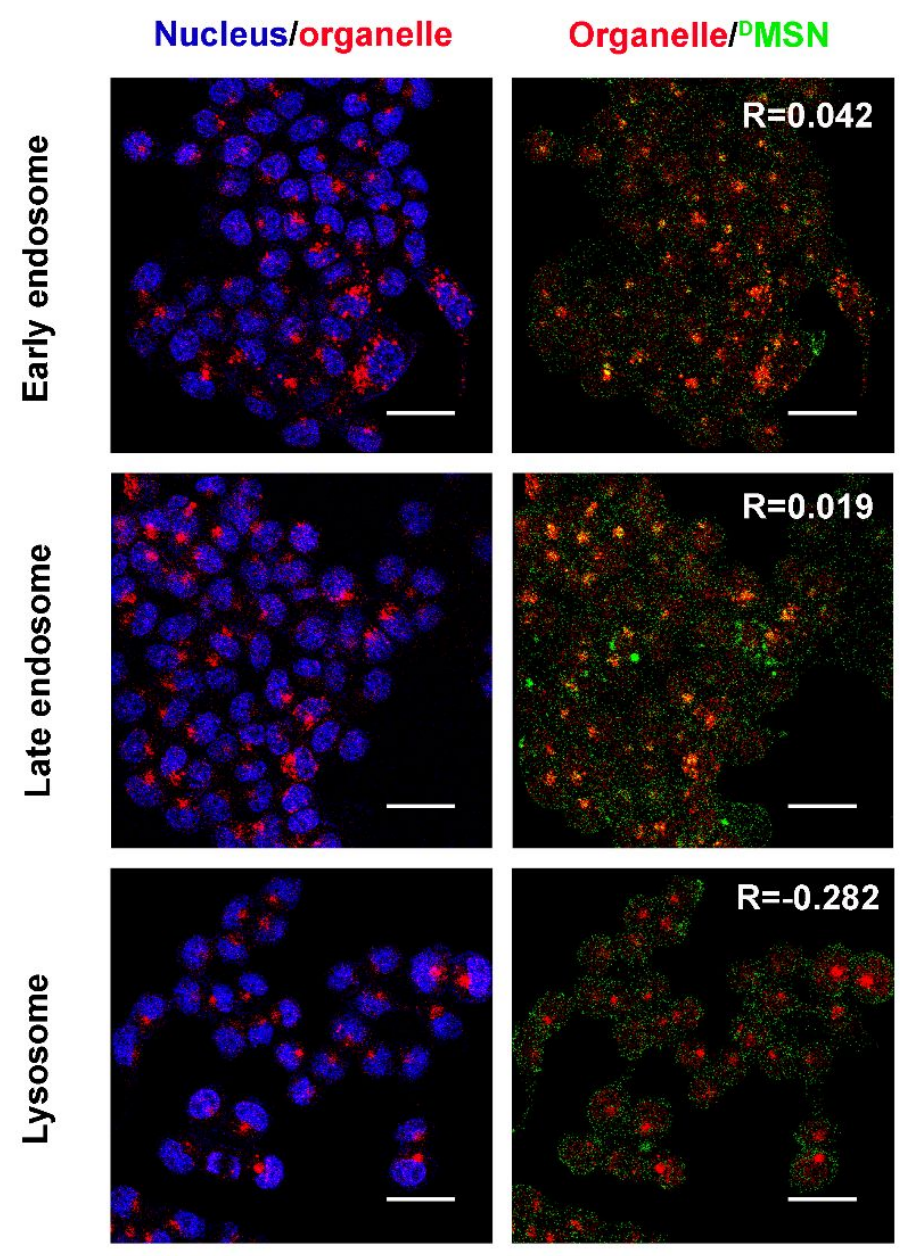

Figure S6. Evaluation of co-localization of ${ }^{\mathrm{D} M S N}$ with lysosome, early and late endosomes. The subcellular organelles were marked in red, and the R stands for Pearson product-moment correlation coefficient. (scale bar: $50 \mu \mathrm{m}$ ) 


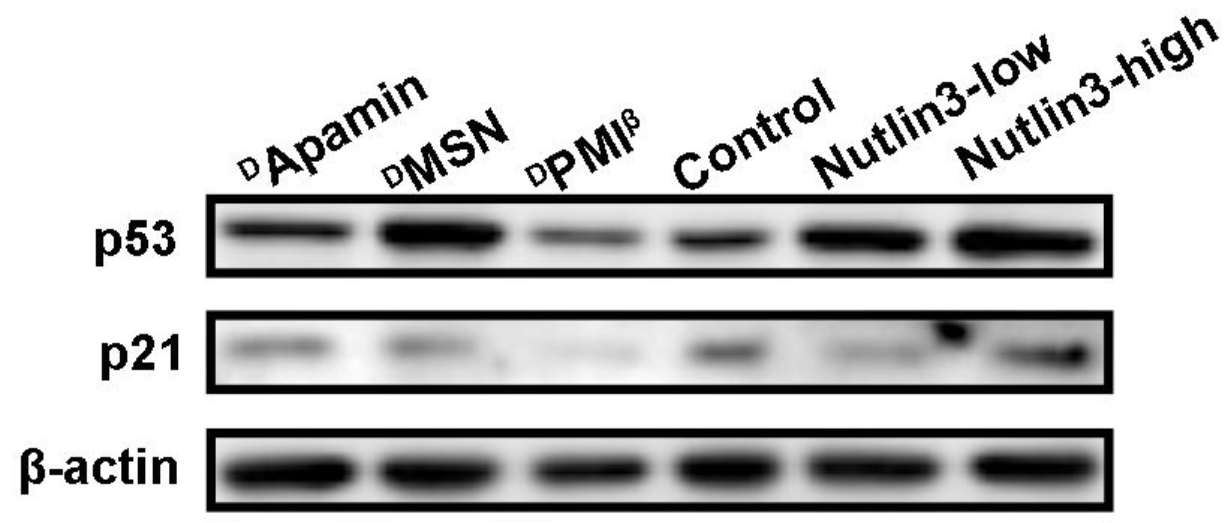

Figure S7. Western blot analysis to monitor the change in protein expression of p53 and p21 in HCT116 cells with the $24 \mathrm{~h}$ treatments of ${ }^{\mathrm{D} M S N}(2 \mu \mathrm{M})$, ${ }^{\mathrm{DPMI}}{ }^{\beta}(2 \mu \mathrm{M})$, ${ }^{\mathrm{D}}$ Apamin $(2 \mu \mathrm{M})$, lowconcentration Nutlin3 $(2 \mu \mathrm{M})$ or high-concentration Nutlin3 $(6 \mu \mathrm{M})$. $\beta$-Actin was used as an internal reference. 
A

DMSN

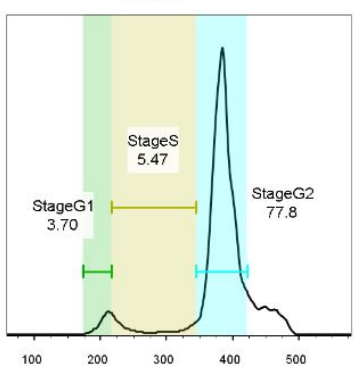

D

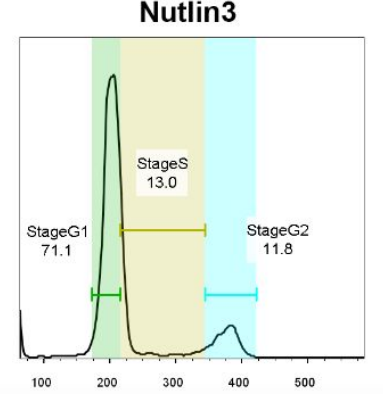

B

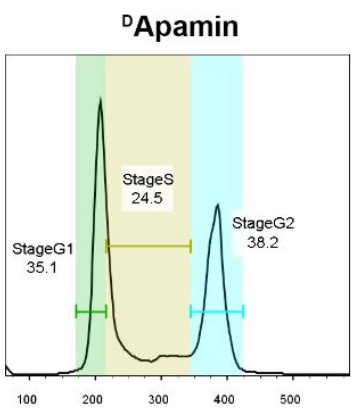

$\mathbf{E}$

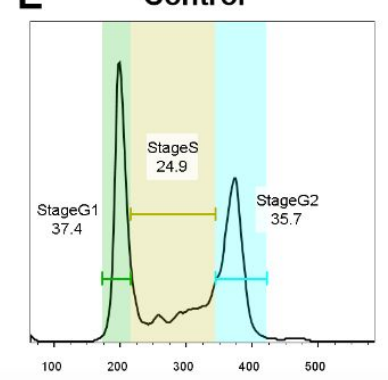

C

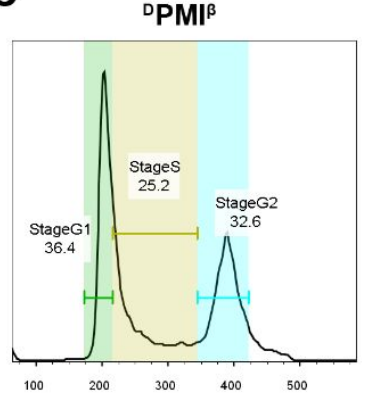

$\mathbf{F}$

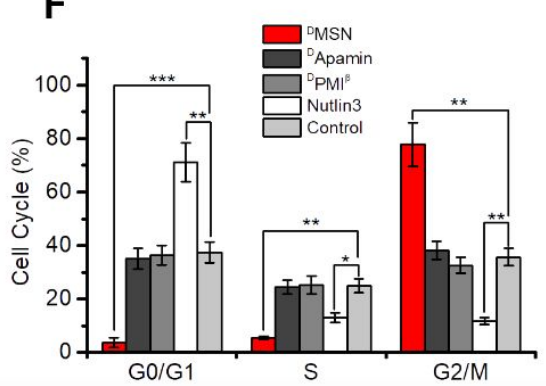

Figure S8. Cell cycle analysis of HCT116 p53 $3^{+/+}$cells after ${ }^{D}$ MSN, ${ }^{D}$ Apamin, ${ }^{D}$ PMI $^{\beta}$ or Nutlin-3 treatment. A-E, HCT116 cells were treated with $2 \mu \mathrm{M}{ }^{\mathrm{D}} \mathrm{MSN}$ (A), and, $2 \mu \mathrm{M}^{\mathrm{D}} \mathrm{Apamin}(\mathrm{B}), 2 \mu \mathrm{M}$ ${ }^{D P M I}{ }^{\beta}(C), 2 \mu \mathrm{M}$ Nutlin-3 (D) or blank control (E) for $24 \mathrm{~h}$, and cell cycle distributions were analyzed by flow cytometry of the PI fluorescence signal. F, statistical analysis of the cell cycle after treatments. The data were presented as mean \pm s.d. of values from three independent experiments. $p$ values were calculated by t-test $\left(*, p<0.05 ; *^{*}, p<0.01 ; * * *, p<0.001\right)$ 

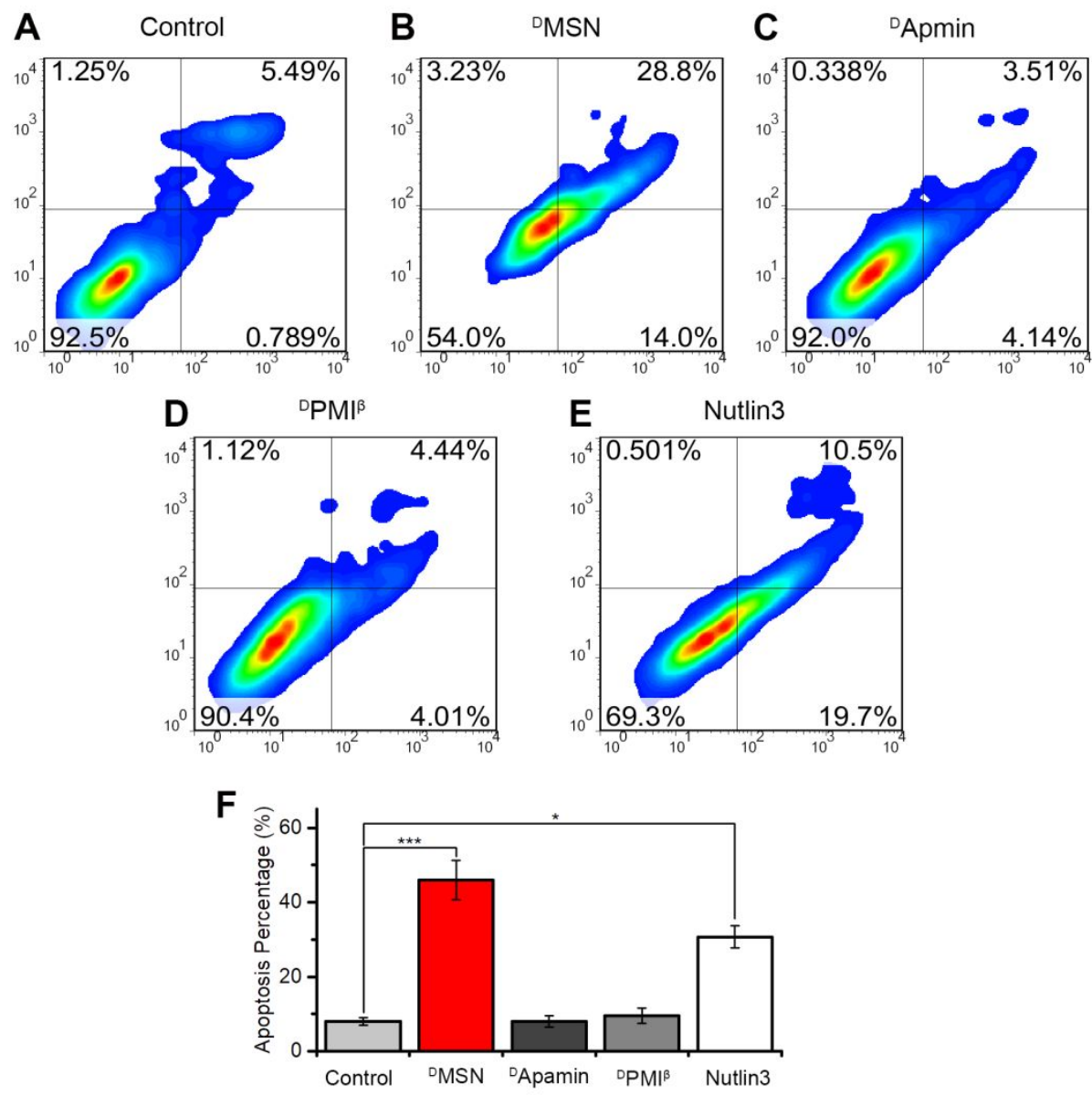

Figure S9. Cell apoptosis analysis of HCT116 $\mathrm{p}^{3}{ }^{+/+}$cells after ${ }^{\mathrm{D} M S N},{ }^{\mathrm{D}} \mathrm{Apamin},{ }^{\mathrm{D} P M{ }^{\beta}}$ or Nutlin-3 treatment. A, the blank control of HCT116 $\mathrm{p}^{23^{+/+}}$cells. B-E, cells were treated with $2 \mu \mathrm{M}{ }^{\mathrm{D}} \mathrm{MSN}$ (B), $2 \mu \mathrm{M}^{\mathrm{D}}$ Apamin (C), $2 \mu \mathrm{M}^{\mathrm{DPMMI}}{ }^{\beta}$ (D) and $2 \mu \mathrm{M}$ Nutlin-3 (E) for $48 \mathrm{~h}$, and cell apoptosis was evaluated by Annexin V-PI staining (abscissa: Annexin V; ordinate: PI) and flow cytometric analysis. F, statistical analysis of the apoptosis after treatments. The data were presented as mean \pm s.d. of values from three independent experiments. $p$ values were calculated by t-test $(*, p<0.05 ; * *$, $p<0.01 ; * * *, p<0.001)$ 


\section{TUNEL}

\section{Control}

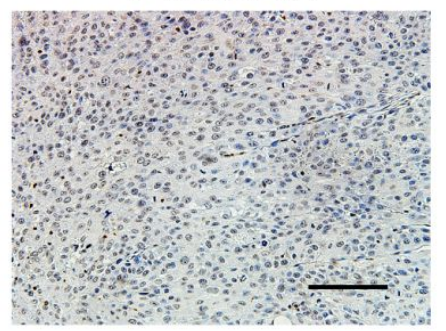

${ }^{\mathrm{D} A p a m i n}$

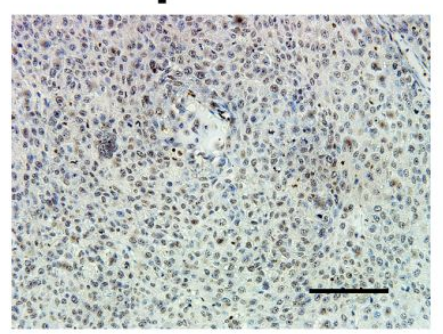

DMSN

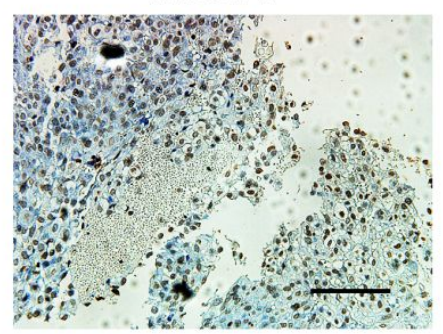

Figure S10. TUNEL staining of representative tumor sections from mice bearing PDX tumor with the indicated treatments (scale bar: $50 \mu \mathrm{m}$ ). 

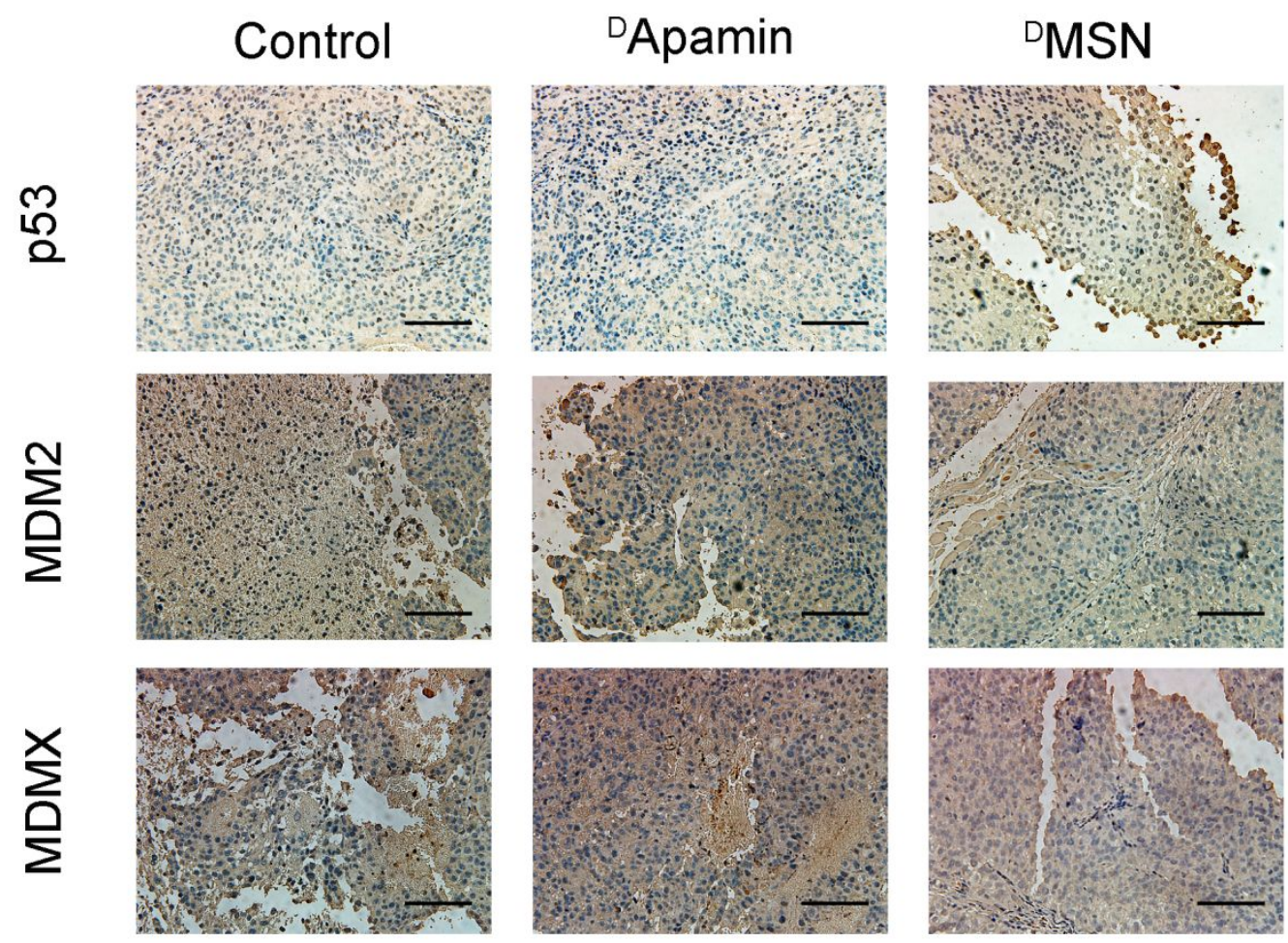

Figure S11. p53, MDM2 and MDMX immunohistochemical staining of representative tumor sections from mice bearing PDX tumor with the indicated treatments (scale bar: $50 \mu \mathrm{m}$ ). 

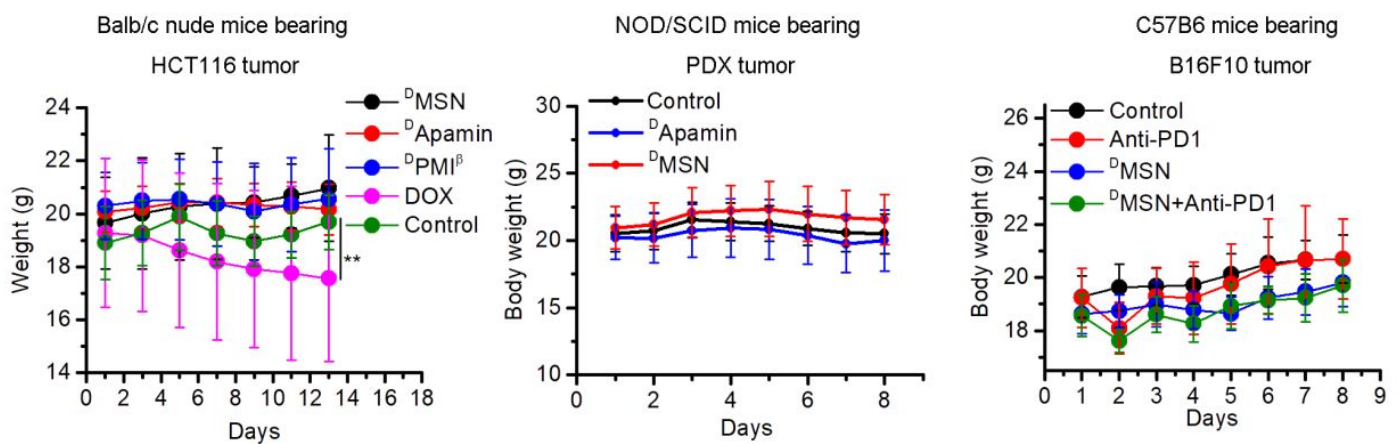

Figure S12. Weight of Balb/c nude mice bearing HCT116 tumor, NOD/SCID mice bearing PDX tumor and C57B6 mice bearing B16F10 tumor with the indicated treatments. The data were presented as mean \pm s.d. of values from three independent experiments. $p$ values were calculated by t-test $(*, p<0.05 ; * *, p<0.01 ; * * *, p<0.001)$ 

A

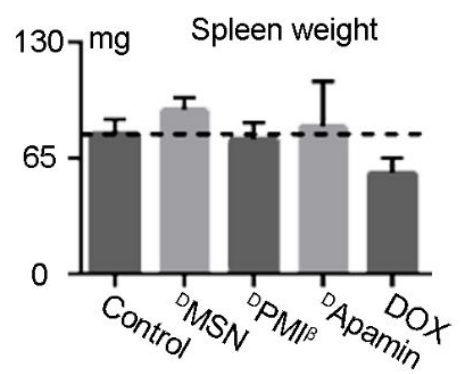

B $H \& E$ of Spleen

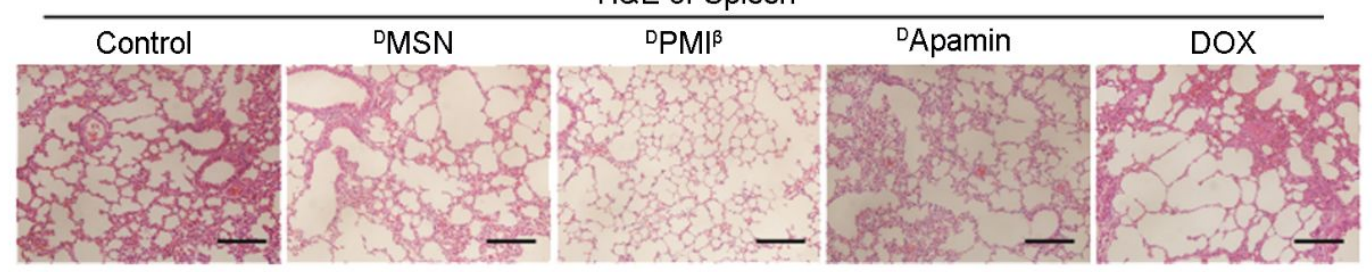

Figure S13. Toxicity evaluation of the Spleen in mice with different treatments. (A) The weight of spleen in mice with the indicated treatments. (B) The representative H\&E staining of heart sections in mice with the indicated treatments (scale bar: $50 \mu \mathrm{m}$ ). 
A

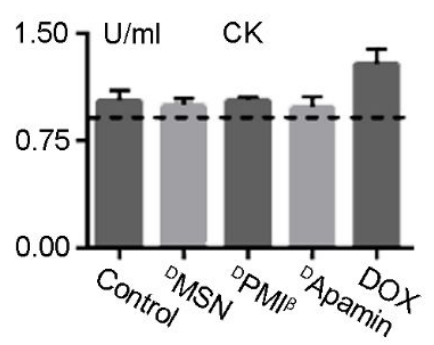

B

H\&E of Heart

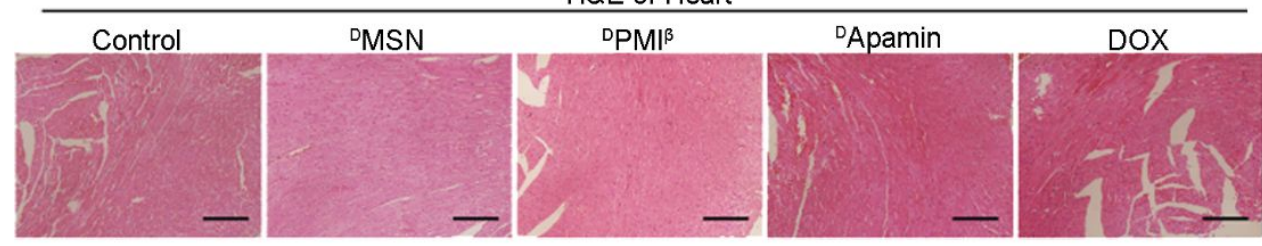

Figure S14. Toxicity evaluation of the Heart in mice with different treatments. (A) The activity of serum creatine kinase $(\mathrm{CK})$ in mice with the indicated treatments. Clinically, $\mathrm{CK}$ is assayed in blood tests as a marker of damage of CK-rich tissues such as in myocardial infarction (heart attack). $p$ values were calculated by $t$-test (unmarked $p>0.05$ ). (B) The representative H\&E staining of heart sections in mice with the indicated treatments (scale bar: $50 \mu \mathrm{m}$ ). 

H\&E of Lung

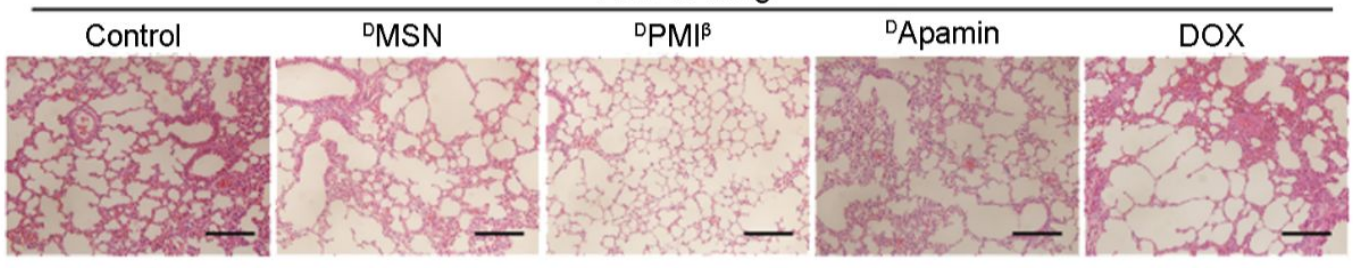

Figure S15. Toxicity evaluation of the lung in mice with different treatments. The representative $H \& E$ staining of lung tissues in mice with the indicated treatments (scale bar: $50 \mu \mathrm{m})$. 


\section{Reference in Supplementary}

[1] C. Li, M. Pazgier, M. Liu, W. Y. Lu, W. Lu, Angewandte Chemie International Edition $2009,48,8712-8715$. 